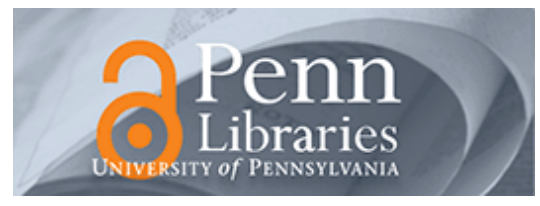

University of Pennsylvania

ScholarlyCommons

$3-6-2008$

\title{
Superconducting Proximity Effect and Majorana Fermions at the Surface of a Topological Insulator
}

\author{
Liang Fu \\ University of Pennsylvania \\ Charles L. Kane \\ University of Pennsylvania, kane@physics.upenn.edu
}

Follow this and additional works at: https://repository.upenn.edu/physics_papers

Part of the Physics Commons

\section{Recommended Citation}

Fu, L., \& Kane, C. L. (2008). Superconducting Proximity Effect and Majorana Fermions at the Surface of a Topological Insulator. Retrieved from https://repository.upenn.edu/physics_papers/99

Suggested Citation:

L. Fu and C.L. Kane. (2008). "Superconducting Proximity Effect and Majorana Fermions at the Surface of a Topological Insulator." Physical Review Letters. 100, 096407.

(c) 2008 The American Physical Society

http://dx.doi.org/10.1103/PhysRevLett.100.096407

This paper is posted at ScholarlyCommons. https://repository.upenn.edu/physics_papers/99

For more information, please contact repository@pobox.upenn.edu. 


\title{
Superconducting Proximity Effect and Majorana Fermions at the Surface of a Topological Insulator
}

\author{
Abstract \\ We study the proximity effect between an s-wave superconductor and the surface states of a strong \\ topological insulator. The resulting two-dimensional state resembles a spinless $p_{x}+i p_{y}$ superconductor, \\ but does not break time reversal symmetry. This state supports Majorana bound states at vortices. We \\ show that linear junctions between superconductors mediated by the topological insulator form a \\ nonchiral one-dimensional wire for Majorana fermions, and that circuits formed from these junctions \\ provide a method for creating, manipulating, and fusing Majorana bound states.

\section{Disciplines} \\ Physical Sciences and Mathematics | Physics

\section{Comments} \\ Suggested Citation: \\ L. Fu and C.L. Kane. (2008). "Superconducting Proximity Effect and Majorana Fermions at the Surface of a \\ Topological Insulator." Physical Review Letters. 100, 096407. \\ (c) 2008 The American Physical Society \\ http://dx.doi.org/10.1103/PhysRevLett.100.096407
}




\title{
Superconducting Proximity Effect and Majorana Fermions at the Surface of a Topological Insulator
}

\author{
Liang Fu and C. L. Kane \\ Department of Physics and Astronomy, University of Pennsylvania, Philadelphia, Pennsylvania 19104, USA
}

(Received 11 July 2007; published 6 March 2008)

\begin{abstract}
We study the proximity effect between an $s$-wave superconductor and the surface states of a strong topological insulator. The resulting two-dimensional state resembles a spinless $p_{x}+i p_{y}$ superconductor, but does not break time reversal symmetry. This state supports Majorana bound states at vortices. We show that linear junctions between superconductors mediated by the topological insulator form a nonchiral one-dimensional wire for Majorana fermions, and that circuits formed from these junctions provide a method for creating, manipulating, and fusing Majorana bound states.
\end{abstract}

DOI: 10.1103/PhysRevLett.100.096407

Excitations with non-Abelian statistics [1] are the basis for the intriguing proposal of topological quantum computation [2]. The simplest non-Abelian excitation is the zero energy Majorana bound state (MBS) associated with a vortex in a spinless $p_{x}+i p_{y}$ superconductor [3-6]. The presence of $2 N$ vortices leads to a $2^{N}$-fold ground state degeneracy. Braiding processes, in which the vortices are adiabatically rearranged, perform nontrivial operations in that degenerate space. Though MBSs do not have the structure necessary to construct a universal quantum computer [7], the quantum information encoded in their degenerate states is topologically protected from local sources of decoherence [8].

MBSs have been proposed to exist as quasiparticle excitations of the $\nu=5 / 2$ quantum Hall effect [1,3], in the cores of $h / 4 e$ vortices in the $p$-wave superconductor $\mathrm{Sr}_{2} \mathrm{RuO}_{4}$ [9] and in cold atoms [10,11]. In this Letter we show that the proximity effect between an ordinary $s$-wave superconductor and the surface of a strong topological insulator (TI) [12-15] leads to a state that hosts MBSs at vortices. We then show that a linear superconductorTI-superconductor (STIS) junction forms a nonchiral 1D wire for Majorana fermions. Such junctions can be combined into circuits, which allow for the creation, manipulation, and fusion of MBSs.

A strong TI is a material with an insulating time reversal invariant band structure for which strong spin orbit interactions lead to an inversion of the band gap at an odd number of time reversed pairs of points in the Brillouin zone. Candidate materials include the semiconducting alloy $\mathrm{Bi}_{1-x} \mathrm{Sb}_{x}$, as well as $\mathrm{HgTe}$ and $\alpha$-Sn under uniaxial strain [15]. Strong TIs are distinguished from ordinary insulators by the presence of surface states, whose Fermi arc encloses an odd number of Dirac points and is associated with a Berry's phase of $\pi$. In the simplest case, there is a single nondegenerate Fermi arc described by the time reversal invariant Hamiltonian

$$
H_{0}=\psi^{\dagger}(-i v \vec{\sigma} \cdot \nabla-\mu) \psi
$$

Here $\psi=\left(\psi_{\uparrow}, \psi_{\downarrow}\right)^{T}$ are electron field operators, $\vec{\sigma}=$
PACS numbers: 71.10.Pm, 03.67.Lx, 74.45.+c, 74.90.+n

$\left(\sigma^{x}, \sigma^{y}\right)$ are Pauli spin matrices, and $\mu$ is the chemical potential. $H_{0}$ can only exist on a surface because it violates the fermion doubling theorem [16]. The topological metal is essentially half of an ordinary $2 \mathrm{D}$ electron gas.

Suppose that an $s$-wave superconductor is deposited on the surface. Because of the proximity effect, Cooper pairs can tunnel into the surface states. This can be described by adding $V=\Delta \psi_{\uparrow}^{\dagger} \psi_{\downarrow}^{\dagger}+$ H.c. to $H_{0}$, where $\Delta=\Delta_{0} e^{i \phi}$ depends on the phase $\phi$ of the superconductor and the nature of the interface [17]. The states of the surface can then be described by $H=\Psi^{\dagger} \mathcal{H} \Psi / 2$, where in the Nambu notation $\Psi=\left(\left(\psi_{\uparrow}, \psi_{\downarrow}\right),\left(\psi_{\downarrow}^{\dagger},-\psi_{\uparrow}^{\dagger}\right)\right)^{T}$ and

$$
\mathcal{H}=-i v \tau^{z} \sigma \cdot \nabla-\mu \tau^{z}+\Delta_{0}\left(\tau^{x} \cos \phi+\tau^{y} \sin \phi\right) .
$$

$\vec{\tau}$ are Pauli matrices that mix the $\psi$ and $\psi^{\dagger}$ blocks of $\Psi$. Time reversal invariance follows from $[\Theta, \mathcal{H}]=0$, where $\Theta=i \sigma^{y} K$ and $K$ is complex conjugation. Particle hole symmetry is expressed by $\Xi=\sigma^{y} \tau^{y} K$, which satisfies $\{\Xi, \mathcal{H}\}=0$. When $\Delta$ is spatially homogeneous, the excitation spectrum is $E_{\mathbf{k}}= \pm \sqrt{( \pm v|\mathbf{k}|-\mu)^{2}+\Delta_{0}^{2}}$. For $\mu \gg \Delta_{0}$, the low energy spectrum resembles that of a spinless $p_{x}+i p_{y}$ superconductor. This analogy can be made precise by defining $c_{\mathbf{k}}=\left(\psi_{\uparrow \mathbf{k}}+e^{i \theta_{\mathbf{k}}} \psi_{\backslash \mathbf{k}}\right) / \sqrt{2}$ for $\mathbf{k}=k_{0}\left(\cos \theta_{\mathbf{k}}, \sin \theta_{\mathbf{k}}\right)$ and $v k_{0} \sim \mu$. The projected Hamiltonian is then $\sum_{\mathbf{k}}(v|\mathbf{k}|-\mu) c_{\mathbf{k}}^{\dagger} c_{\mathbf{k}}+\left(\Delta e^{i \theta_{\mathbf{k}}} c_{\mathbf{k}}^{\dagger} c_{-\mathbf{k}}^{\dagger}+\right.$ H.c.)/2. Though this is formally equivalent to a spinless $p_{x}+i p_{y}$ superconductor, there is an important difference: $\mathcal{H}$ respects time reversal symmetry, while the $p_{x}+i p_{y}$ superconductor does not.

It is well known that a $h / 2 e$ vortex in a $p_{x}+i p_{y}$ superconductor leads to a MBS [3]. This suggests that for $\mu \gg \Delta_{0}$ a similar bound state should exist for (2). The bound states at a vortex are determined by solving the Bogoliubov-de Gennes (BdG) equation $\mathcal{H} \xi=E \xi$ in polar coordinates with $\Delta(r, \theta)=\Delta_{0}(r) e^{ \pm i \theta}$. A zero energy solution exists for any $\mu$. The algebra is simplest for $\mu=$ 0 , where the zero mode has the form 


$$
\xi_{0}^{ \pm}(r, \theta)=\chi^{ \pm} e^{-\int_{0}^{r} d r^{\prime} \Delta_{0}\left(r^{\prime}\right) / v},
$$

with $\chi^{+}=((0, i),(1,0))^{T}$ and $\chi^{-}=((1,0),(0,-i))^{T}$.

Another feature of $p_{x}+i p_{y}$ superconductors is the presence of chiral edge states $[3,18,19]$. With time reversal symmetry, chiral edge states cannot occur in our system. The surface - which itself is the boundary of a threedimensional crystal - cannot have a boundary. By breaking time reversal symmetry, however, a Zeeman field can introduce a mass term $M \sigma^{z}$ into (1) and (2), which can open an insulating gap in the surface state spectrum. By solving (2) we find that the interface between this insulating state and the superconducting state has chiral Majorana edge states. This could possibly be realized by depositing superconducting and insulating magnetic materials on the surface to form a superconductor-TI-magnet junction. It is interesting to note that for spinless electrons the $p_{x}+i p_{y}$ superconductor violates time reversal, while the vacuum does not. For our surface states it is the insulator that violates time reversal. A related effect could also occur at the edge of a two-dimensional TI [20-22], which is described by (1) and (2) restricted to one spatial dimension. At the boundary between a region with superconducting gap $\Delta \tau^{x}$ and a region with insulating gap $M \sigma^{z}$ we find a MBS, analogous to the end states discussed in Refs. [23,24]. In the following we will focus on STIS junctions, which can lead to nonchiral one-dimensional Majorana fermions, as well as MBSs.

Consider a line junction of width $W$ and length $L \rightarrow \infty$ between two superconductors with phases 0 and $\phi$ in contact with TI surface states. We analyze the Andreev bound states in the surface state channel between the superconductors by solving the $\mathrm{BdG}$ equation with $\Delta(x, y)=\Delta_{0} e^{i \phi}$ for $y>W / 2, \Delta_{0}$ for $y<-W / 2$, and 0 otherwise. The calculation is similar to Titov, Ossipov, and Beenakker's [25] analysis of graphene superconductornormal-superconductor (SNS) junctions, except for the important difference that graphene has four independent Dirac points, while we have only one. For $W \ll v / \Delta_{0}$ there are two branches of bound states, which disperse with the momentum $q$ in the $x$ direction. For $W=\mu=0$ we find

$$
E_{ \pm}(q)= \pm\left[v^{2} q^{2}+\Delta_{0}^{2} \cos ^{2}(\phi / 2)\right]^{1 / 2} .
$$

For $\phi=\pi$ the spectrum is gapless. It is useful to construct a low energy theory, for $q \sim 0$ and $\phi=\pi-\epsilon$. Finite $W$ and $\mu$ can then easily be included. We first solve the BdG equation for the two $E=0$ modes $\zeta_{a=1,2}(y)$ at $q=0$ and $\phi=\pi$. It is useful to choose them to satisfy $\Xi \zeta_{a}=\zeta_{a}$. Up to a normalization they may be written

$$
\zeta_{1} \pm i \zeta_{2}=((1, \pm i),( \pm i,-1))^{T} e^{ \pm i \mu y / v-\int_{0}^{|y|} d \tilde{y} \Delta_{0}(\tilde{y}) / v} .
$$

We next evaluate $\left\langle\zeta_{a}\left|q \sigma^{x} \tau^{z}\right| \zeta_{b}\right\rangle$ and $\left\langle\zeta_{a}\right| \epsilon \Delta_{0} \theta(y-$ $W) \tau^{y}\left|\zeta_{b}\right\rangle$ to obtain the " $k \cdot p$ " Hamiltonian,

$$
\tilde{\mathcal{H}}=-i \tilde{v} \tau^{x} \partial_{x}+\delta \tau^{y},
$$

where $\quad \tilde{v}=v\left[\cos \mu W+\left(\Delta_{0} / \mu\right) \sin \mu W\right] \Delta_{0}^{2} /\left(\mu^{2}+\Delta_{0}^{2}\right)$ and $\delta=\Delta_{0} \cos (\phi / 2)$. The Pauli matrices $\tau_{a b}^{x, y}$ act on $\zeta_{a}$ and are different from those in (2). In this basis $\Theta=i \tau^{y} K$ and $\Xi=K . \tilde{\mathcal{H}}$ resembles the Su-Schrieffer-Heeger (SSH) model [26]. However, unlike that model, the $E_{ \pm}(q)$ states are not independent, and the corresponding Bogoliubov quasiparticle operators satisfy $\gamma_{+}(q)=\gamma_{-}(-q)^{\dagger}$. The system is thus half a regular 1D Fermi gas, or a nonchiral "Majorana quantum wire."

Below it will be useful to consider junctions that bend and close. When a line junction makes an angle $\theta$ with the $x$ axis, the basis vectors (5) are modified according to $\zeta_{a} \rightarrow$ $e^{i \sigma_{z} \theta / 2} \zeta_{a} . \tilde{\mathcal{H}}$, however, is unchanged even when $\theta(x)$ varies. On a circle, $\zeta_{a}$ changes sign when $\theta$ advances by $2 \pi$. Therefore, eigenstates of $\tilde{\mathcal{H}}$ must obey antiperiodic boundary conditions, $\varphi(0)=-\varphi(2 \pi)$.

Next consider a trijunction, where three superconductors separated by line junctions meet at a point, as in Fig. 1(c). When $\phi_{k=1,2}$ is in the shaded region of Fig. 1(d), a MBS exists at the junction. Though the general BdG equation cannot be solved analytically, this phase diagram can be deduced by solving special limits. When $\phi_{k}=0$, there is no bound state. Another solvable limit is when three line junctions with $W=0$ are oriented at $120^{\circ}$, and $\phi_{k}=$ $\pm k(2 \pi / 3)$. This is a discrete analog of a \pm vortex with $C_{3}$ symmetry, and is indicated by the circles in Fig. 1(d). For $\mu=0$ we find a MBS identical to (3) with the exponent replaced by $-\Delta_{0} \hat{n} \cdot \mathbf{r} / v$. Here $\hat{n}$ is a constant unit vector in each superconductor that bisects the angle between neighboring junctions. The MBS cannot disappear when $\phi_{k}$ are changed continuously unless the energy gap closes. The phase boundaries indicated in Fig. 1(d) therefore follow from the solution of the line junction and occur
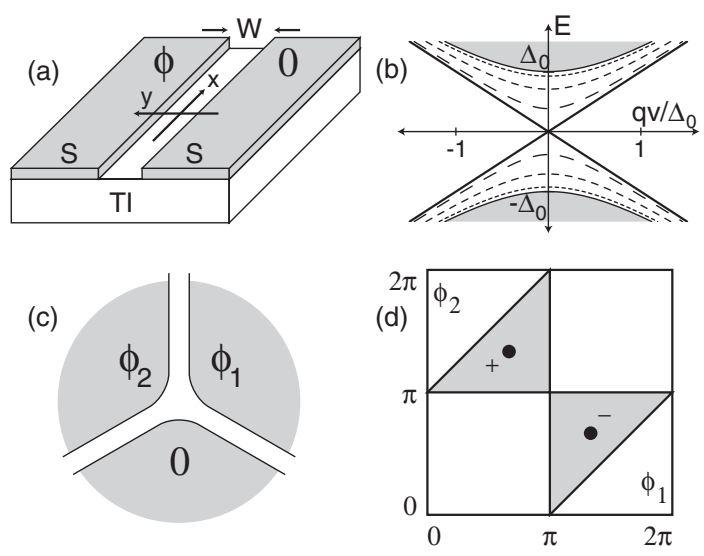

FIG. 1. (a) A STIS line junction. (b) Spectrum of a line junction for $W=\mu=0$ as a function of momentum for various $\phi$. The solid line shows the Andreev bound states for $\phi=\pi$. The dashed lines are for $\phi=3 \pi / 4, \pi / 2$, and $\pi / 4$. The bound states for $\phi=0$ merge with the continuum, indicated by the shaded region. (c) A trijunction between three superconductors. (d) Phase diagram for the trijunction. In the shaded regions there is a \pm MBS at the junction. 
when the phase difference between neighboring superconductors is $\pi$.

It is instructive to consider the limit where two of the lines entering the trijunction are nearly gapless. For $\phi_{1}=$ $\pi-\epsilon_{1}$ and $\phi_{2}=\pi-\epsilon_{2}$ Fig. 1(d) predicts a MBS when $\epsilon_{1} \epsilon_{2}<0$. This can be understood with Eq. (6), which describes the lower two line junctions, which have masses $\delta_{1,2}=\Delta_{0} \epsilon_{1,2} / 2$. When $\epsilon_{1} \epsilon_{2}<0, \delta$ changes sign, leading to the well known midgap state of the SSH model [26,27], which in the present context is a MBS.

A line junction terminated by two trijunctions allows MBSs to be created, manipulated, and fused. When $\phi$ passes through $\pi$, MBSs appear or disappear at both ends. To model this, we assume the phases of the superconductors on either side of the line junction are 0 and $\pi-$ $\epsilon$, and that the superconductors at the left (right) ends have phases $\phi_{L(R)}$, which are not close to 0 or $\pi$. This allows us to model the ends using a hard wall boundary condition $\delta \rightarrow \pm \infty$, where the sign at each end is $s_{L, R}=$ $\operatorname{sgn} \sin \phi_{L, R}$. It is straightforward to solve (6) to determine the spectrum as a function of $\delta=\Delta_{0} \epsilon / 2$ for a line of length $L$ using this boundary condition. There are two cases depending on the sign of $s_{L} s_{R}$.

For $s_{L}=s_{R}=1$ either zero or a \pm pair of MBSs are expected. The spectrum, shown in Fig. 2(a), may be written $E_{n}^{ \pm}(\delta)= \pm \sqrt{\delta^{2}+\tilde{v}^{2} k_{n}^{2}}$, where $k_{n}$ are solutions to $\tan k_{n} L=-\tilde{v} k_{n} / \delta$. Midgap states are present for $\delta<0$. For $L \rightarrow \infty$ a pair of zero energy states $\xi_{1,2}(x, y)$ are localized at each end with wave functions

$$
\xi_{1}=\zeta_{1} e^{-|\delta| x / \tilde{v}}, \quad \xi_{2}=\zeta_{2} e^{-|\delta|(L-x) / \tilde{v}},
$$

where $\zeta_{a}(y)$ are given in (5). For finite $-\delta L / \tilde{v} \gg 1$ the eigenstates are $\varphi_{ \pm}=\xi_{1} \pm i \xi_{2}$, with energies $E_{0}^{ \pm}(\delta) \sim$ $\pm 2|\delta| e^{-|\delta| L / \tilde{v}}$. These define Bogoliubov quasiparticle operators, $\Gamma_{ \pm}=\left(\varphi_{ \pm}\right)^{\dagger} \Psi$. Since $\varphi_{ \pm}=\Xi \varphi_{\mp}$, it follows that $\Gamma_{+}=\Gamma_{-}^{\dagger} \equiv\left(\gamma_{1}-i \gamma_{2}\right) / 2$ where $\gamma_{a}=\left(\xi_{a}\right)^{\dagger} \Psi \quad$ are Majorana operators. The pair $\gamma_{1,2}$ thus defines a two state Hilbert space indexed by $n_{12}=\Gamma_{+}^{\dagger} \Gamma_{+}$. The splitting between $\varphi_{ \pm}$then characterizes the interaction between the MBSs,
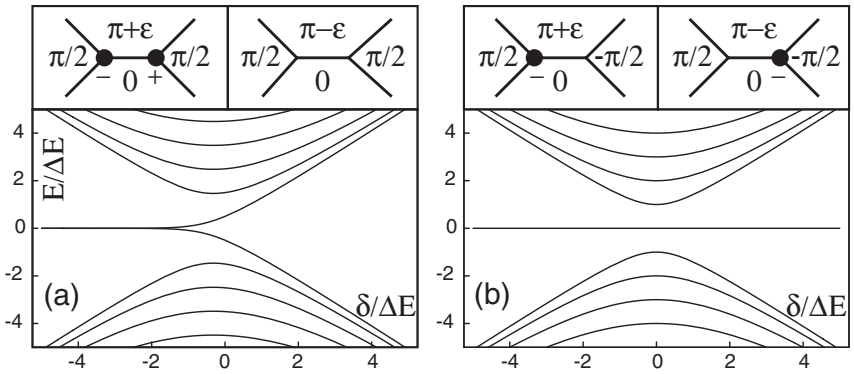

FIG. 2. Energy levels in units of $\Delta E=\tilde{v} / L$ for a STIS line junction terminated by two trijunctions as a function of $\delta=$ $\Delta_{0} \cos \phi / 2$ for $\phi \sim \pi$. In (a) two MBSs are created or fused when $\phi$ passes through $\pi$. In (b) a single MBS is transported from one end to the other. The insets depict the MBSs.

$$
H=E_{0}^{+}(\delta)\left(\Gamma_{+}^{\dagger} \Gamma_{+}-1 / 2\right)=i E_{0}^{+}(\delta) \gamma_{2} \gamma_{1} / 2 .
$$

The $s_{L}=s_{R}=-1$ case is similar. Equation (8) applies to both cases, provided $\gamma_{2}$ is associated with the + vortex.

This provides a method for both creating and fusing pairs of MBSs. Suppose we begin in the ground state at $\delta>0$ with no MBSs present. Upon adiabatically decreasing $\delta$ through 0 , MBSs appear in the state $\left|0_{12}\right\rangle$. Next suppose that initially $\delta<0$, and a pair of MBSs are present in the state $\left|n_{12}\right\rangle$. When $\delta$ is adiabatically increased through 0 , the system will remain in $\left|n_{12}\right\rangle$, which will either evolve to the ground state or to a state with one extra fermion. The difference between the two states can be probed by measuring the current flowing across the linear junction, which depends on whether the Andreev bound state $\varphi_{+}$is occupied. The measured current will be $I=$ $\bar{I} \pm \Delta I / 2$, where the current carried by $\varphi_{+}$is $\Delta I=$ $(e / \hbar) d E_{0}^{+} / d \phi \sim e \Delta_{0} / 2 \hbar$ for $\delta L / \tilde{v} \gtrsim 1$. For $\Delta_{0} \sim$ $0.1 \mathrm{meV}, \Delta I \sim 10 \mathrm{nA}$.

Finally, consider the case $s_{L}=-s_{R}=1$, in which a - MBS is at one end or the other, as in Fig. 2(b). There are plane wave solutions with energy $E_{n}^{ \pm}=$ $\pm \sqrt{\delta^{2}+(n \pi \tilde{v} / L)^{2}}$ for $n=1,2, \ldots$, along with a single $E_{0}=0$ state with wave function

$$
\xi(x, y) \propto \zeta_{1}(y) e^{\delta x / \tilde{v}} .
$$

Depending on the sign of $\delta, \xi$ is exponentially localized at one end or the other. When $\delta$ changes sign, the MBS smoothly switches sides. This provides a method for transporting a MBS from one node to another.

We now discuss simple circuits built from STIS junctions. First, consider Fig. 3(a) and a process in which the phase of the central island is adiabatically advanced from 0 to $2 \pi$. For $\phi=0$ there are no MBSs. At $\phi=2 \pi / 3$ two pairs of MBSs are created at the top and bottom line junctions. At $\phi=4 \pi / 3$ the MBSs are fused at the left and right line junctions. If the system begins in the ground state $\phi=0$, then when $\phi \rightarrow 2 \pi$ we find [8]

$$
\left|0_{12} 0_{34}\right\rangle \rightarrow\left(\left|0_{14} 0_{32}\right\rangle+\left|1_{14} 1_{32}\right\rangle\right) / \sqrt{2} \text {. }
$$

Thus, after the cycle, the left and right segments are in an entangled state. The currents measured across the left and right junctions will be $\bar{I} \pm \Delta I / 2$ with $50 \%$ probability and will be perfectly correlated.

Equation (10) can be understood in two ways. First, the cycle effectively creates two pairs of MBS's, interchanges a pair (say, 2 and 4), and brings the pairs back together. As shown by Ivanov [4], this corresponds to the operator $P_{24}=\left(1+\gamma_{2} \gamma_{4}\right) / \sqrt{2}$, which leads directly to $(10)$. Alternatively, this result can be derived from (6) and (8). From (8), the Hamiltonian for $\phi \leqq 2 \pi / 3$ is $H_{1} \propto$ $i\left(\gamma_{1} \gamma_{2}+\gamma_{3} \gamma_{4}\right)$. For $\phi \geq 4 \pi / 3$ it becomes $H_{2} \propto$ $i\left(\gamma_{1} \gamma_{4}-\gamma_{3} \gamma_{2}\right)$. Here the minus sign arises because, as explained after Eq. (6), the closed 1D circuit must have antiperiodic boundary conditions. Thus, one of the line junctions must have a cut where the wave function changes 

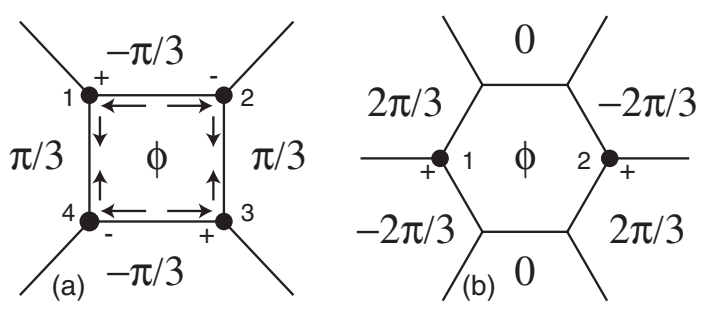

FIG. 3. Simple circuits made from STIS junctions. When $\phi$ is advanced from 0 to $2 \pi$, (a) produces an entangled state and (b) interchanges MBSs 1 and 2.

sign. We chose the cut to be on the junction between 2 and 3. It is then straightforward to express the ground state of $H_{1}$ in terms of the eigenstates of $H_{2}$, which leads directly to (10).

Figure 3(b) gives a geometry for interchanging MBSs without fusing them. For $\phi=0$ MBSs are located as shown. When $\phi$ advances by $2 \pi$ the MBSs hop counterclockwise 3 times and are interchanged. Ivanov's rules [4] predict $\gamma_{2} \rightarrow \gamma_{1}, \gamma_{1} \rightarrow-\gamma_{2}$. Again the minus sign can be understood in terms of the cut due to antiperiodic boundary conditions. One can imagine larger arrays, where this process performs elementary braiding operations.

The experimental implementation of this proposal will require progress on many fronts. The first is to find a strong TI with a robust gap. $\mathrm{Bi}_{1-x} \mathrm{Sb}_{x}$ and strained $\mathrm{HgTe}$ can have gaps of order $30 \mathrm{meV}$ [15]. The next is to interface with an appropriate superconductor. $\Delta_{0}$ depends on the quality of the interface, Schottky barriers, and the mismatch in the Fermi wavelengths [17]. If these can be optimized, $\Delta_{0}$ can be comparable to the gap of the bulk superconductor [28].

The simplest experimental geometry would be to consider a single line junction with $W \lesssim \hbar v / \Delta_{0}$. For $\Delta_{0} \sim$ $0.1 \mathrm{meV}$ and $\hbar v \sim 1 \mathrm{eV} \AA$, this could be achieved with $W \lesssim 1 \mu \mathrm{m}$. This should be similar to a graphene SNS junction [25]. A signature of the Majorana character of the junction could be probed by measuring the thermal conductance along the channel for $k_{B} T<\Delta_{0}$. For $\phi=\pi$ the central charge $c=1 / 2$ of the gapless Majorana modes leads to a quantized Landauer thermal conductance $\kappa=$ $c\left(\pi^{2} / 3\right)\left(k_{B}^{2} / h\right) T$. By constructing a pair of trijunctions as in Fig. 2 the presence of MBSs can be controlled. It would then be interesting to perform tests of the nonlocality of MBSs envisioned in Refs. [24,29].

Manipulating and fusing MBSs places more stringent requirements on the energy gaps. The junctions should be sufficiently short that $\Delta E=\tilde{v} / L>k_{B} T$, but sufficiently long that the MBSs are well localized. The good news is that $\Delta E$ varies as a power of $L$, while the MBS overlap is exponential, so at low temperature both criteria can be achieved.

If the process of varying $\delta$ to manipulate the MBSs is nonadiabatic or $\Delta E<k_{B} T$, then additional quasiparticles could be excited. If those quasiparticles escape and interact with other MBSs, then the state of the MBSs will be disturbed. However, if $\delta \ll \Delta_{0}$, the excited quasiparticles will be confined to the segment in which they were created. If $\delta$ is turned up so that $k_{B} T \ll \delta \ll \Delta_{0}$, and the system relaxes back to its ground state, then the state of the MBSs will remain intact. Thus, if there is sufficient dynamic range between $k_{B} T$ and $\Delta_{0}$, the system can tolerate these excitations.

We thank Sankar das Sarma and Steve Simon for helpful discussions. This work was supported by NSF Grant No. DMR-0605066, and by ACS PRF Grant No. 44776AC10.

[1] G. Moore and N. Read, Nucl. Phys. B360, 362 (1991).

[2] A. Kitaev, Ann. Phys. (N.Y.) 303, 2 (2003).

[3] N. Read and D. Green, Phys. Rev. B 61, 10267 (2000).

[4] D. A. Ivanov, Phys. Rev. Lett. 86, 268 (2001).

[5] A. Stern, F. von Oppen, and E. Mariani, Phys. Rev. B 70, 205338 (2004).

[6] M. Stone and S. B. Chung, Phys. Rev. B 73, 014505 (2006).

[7] M. H. Freedman, M. Larsen, and Z. Wang, Commun. Math. Phys. 227, 605 (2002).

[8] A. Kitaev, Ann. Phys. (N.Y.) 321, 2 (2006).

[9] S. Das Sarma, C. Nayak, and S. Tewari, Phys. Rev. B 73, 220502(R) (2006).

[10] V. Gurarie, L. Radzihovsky, and A. V. Andreev, Phys. Rev. Lett. 94, 230403 (2005).

[11] S. Tewari et al., Phys. Rev. Lett. 98, 010506 (2007).

[12] L. Fu, C. L. Kane, and E. J. Mele, Phys. Rev. Lett. 98, 106803 (2007).

[13] J.E. Moore and L. Balents, Phys. Rev. B 75, 121306(R) (2007).

[14] R. Roy, arXiv:cond-mat/0607531 (unpublished).

[15] L. Fu and C. L. Kane, Phys. Rev. B 76, 045302 (2007).

[16] H. Nielssen and N. Ninomiya, Phys. Lett. B 130, 389 (1983).

[17] A.F. Volkov et al., Physica (Amsterdam) 242C, 261 (1995).

[18] L. J. Buchholtz and G. Zwicknagl, Phys. Rev. B 23, 5788 (1981).

[19] M. Matsumoto and M. Sigrist, J. Phys. Soc. Jpn. 68, 994 (1999).

[20] C.L. Kane and E. J. Mele, Phys. Rev. Lett. 95, 226801 (2005); 95, 146802 (2005).

[21] S. Murakami, Phys. Rev. Lett. 97, 236805 (2006).

[22] A. Bernevig, T. Hughes, and S. C. Zhang, Science 314, 1757 (2006).

[23] A. Kitaev, arXiv:cond-mat/0010440 (unpublished).

[24] G. W. Semenoff and P. Sodano, arXiv:cond-mat/0601261 (unpublished).

[25] M. Titov and C. W. J. Beenakker, Phys. Rev. B 74, 041401(R) (2006); M. Titov, A. Ossipov, and C. W. J. Beenakker, Phys. Rev. B 75, 045417 (2007).

[26] W. P. Su, J. R. Schrieffer, and A. J. Heeger, Phys. Rev. B 22, 2099 (1980).

[27] R. Jackiw and C. Rebbi, Phys. Rev. D 13, 3398 (1976).

[28] A. Chrestin, T. Matsuyama, and U. Merkt, Phys. Rev. B 55, 8457 (1997).

[29] C. J. Bolech and E. Demler, Phys. Rev. Lett. 98, 237002 (2007). 